\title{
Short timescale UV variability study in NGC 4151 using IUE data
}

\author{
P. VEDAVATHI and VIJAYAKUMAR H. DODDAMANI* \\ Department of Physics, Bangalore University, Bengaluru 560 056, India. \\ *Corresponding author. E-mail: drvkdmani@gmail.com; profvijayakumarhd@bub.ernet.in
}

MS received 24 March 2018; accepted 26 June 2018; published online 27 September 2018

\begin{abstract}
IUE has made very successful long term and intense short time-scale monitoring spectroscopic study of NGC 4151, a Seyfert 1 galaxy for over nearly 18 years from its launch in 1978 to 1996. The long-term observations have been useful in understanding the complex relation between UV continuum and emission line variability Seyfert galaxies. In this paper, we present the results of our studies on the short-timescale intense monitoring campaign of NGC 4151 undertaken during December 1-15, 1993. A most intense monitoring observation of NGC 4151 was carried out by IUE in 1993, when the source was at its historical high flux state with a shortest interval of 70 min between two successive observations. We present our results on emission line and continuum variability amplitudes characterized by $F_{\mathrm{var}}$ method. We found highest variability of nearly $8.3 \%$ at $1325 \AA$ continuum with a smallest amplitude of $4 \%$ at $2725 \AA$. The relative variability amplitudes $\left(R_{\max }\right)$ have been found to be $1.372,1.319,1.302$ and 1.182 at $1325,1475,1655$ and $2725 \AA$ continuum respectively. The continuum and emission line variability characteristics obtained in the present analysis are in very good agreement with the results obtained by Edelson et al. (1996) and Crenshaw et al. (1996) from the analysis of the same observational spectral data. The large amplitude rapid variability characteristics obtained in our study have been attributed to the continuum reprocessing of X-rays absorbed by the material in the accretion disk as proposed by Shakura and Sunyaev (1973). The continuum and emission light curves have shown four distinct high amplitude events of flux maxima during the intense monitoring campaign of 15 days, providing a good limit on the amplitude of UV variability and the BLR size in low luminosity Seyfert galaxies and are useful for constraining the continuum emission models. The decreasing $F_{\text {var }}$ amplitude of UV continuum with respect to increasing wavelength obtained in the present study and consistent with similar observations by Edelson et al. (1996) and Crenshaw et al. (1996) is a significant result of the intense monitoring observations.
\end{abstract}

Keywords. NGC 4151—short-timescale variability—line and continuum fluxes—relative variability amplitude $-F_{\mathrm{var}}$.

\section{Introduction}

Active galaxies exhibit rapid, large amplitude variability in line and continuum fluxes at optical/UV and $\mathrm{X}$-rays. The long-term variability studies of active galaxies have become very useful to probe the spatially unresolved compact nuclear regions and the surrounding broad line region from the light travel time delay calculations between two continuum windows. The broad emission lines such as Ly $\alpha$, Si IV, C IV, He II, CIII] and Mg II lines observed in the UV spectra are among the most prominent features in Seyfert 1 galaxies. The standard picture of the AGN structure that has emerged by now is of the matter accretion on to a compact hot accretion disk surrounding a supermassive black hole. According to the general photoionization models, BEL (Broad Emission Line) region is photo-ionized by the continuum radiation from the central variable source (Netzer 1990; Arav et al. 1998). The spectral variations in the UV and X-ray regions show no simple relationship to set constraints on the possible mechanisms to explain the AGN-variability phenomenon. The continuum and emission line flux variability in Seyfert galaxies have been known since the time of filter photometry, which for the first time reported the variability of the $\mathrm{H}(\alpha)$ line in NGC 4151 (Cherepashchuk \& Lyutyi 1973). The variability studies suggest a chain of cause and effect phenomenon sequentially in that the events are first observed in the thermal continuum components followed by variations 
in the power-law continuum of the form $F_{\lambda} \propto \nu^{\alpha}$ and lastly in the emission line regions. The continuum fades in response to a short fall in the fuel supply from the accretion disc. Active galaxies exhibiting variations in continuum and line fluxes that are characterized by power-law spectra are colored, with less power at higher temporal frequencies (e.g., Gonzalez-Martin and Vaughan 2012). A systematic study of the same would provide information on a range of time-scales and structural stratification of BEL region (or BLR). Our ability to infer the geometry and kinematics of the BEL gas depends very much on the nature of variability, variability amplitude and the time scales involved in the continuum radiation.

In active galaxies, the photo-ionized emission lines respond to changes in the ionizing flux and so, by comparing the associated line and continuum variations, one can measure the size, probe the spatial distribution and velocity field of the emission line gas near the nucleus (Bahcall et al. 1972; Blandford \& McKee 1982; Peterson 1993; Gondhalekar et al. 1994). Therefore, the AGN variability studies have become crucial in exploring the unresolved BLR regions in Seyferts and quasars. The emission line fluxes and velocity profiles that we observe from the nucleus are in fact a superposition of responses from different parts of an extended but unresolved clumpy emission line regions. Line emission from each line site is Doppler-shifted due to the local velocity of the gas and delayed in time because the path from central ionizing source to lineof-sight of emissions to the observer is longer than the direct path of continuum photons from the centre to the observer. The BLR acts as a delay buffer, delivering us line responses that sample the past history of the ionizing flux over a time-scale comparable to the light travel time across the diameter of the BLR. The narrow emission lines in AGN spectra do not appear to vary rapidly because the NLR extends over several hundreds to thousands of parsecs. In contrast, the broad emission line variations on time-scales of days imply that the BLR is of the order of several days in Seyferts. Our ability to infer the geometry and kinematics of the emission line gas depends on the nature of variations in the ionizing flux. An ideal event in the continuum light curve would be a narrow spike resembling a delta function, a large amplitude maximum to minimum with a short time-scale well separated from any previous or following events. Unfortunately, AGNs are hardly ever so co-operative, but their erratic variations do provide information on a range of time-scales which sometimes can be decoded to extract the structural information we seek (Ulrich \& Horne 1996).
In this paper, we present our results on the new spectral analysis of the IUEs most intense monitoring observations of NGC 4151 taken over a period of nearly 15 days during December $1-15,1993$. The IUE satellite launched by NASA in January 1978 has made very successful spectroscopic observations of different kinds of active galaxies such as Sefert galaxies, H II regions, radio galaxies, blazars and quasars (Boggess et al. 1978a,b). These observations are so intense as the minimum time interval between two successive observations is $\sim 70 \mathrm{~min}$ and the closest observations in the UV region ever made for active galaxies. The availability of long-term as well as shortterm UV spectroscopic data of IUE for Seyferts has motivated us to undertake a systematic study of the complete data set for line and continuum variability. However, in this paper we are presenting our results only on short-time scale variability studies only. The NGC 4151 Seyfert galaxy is more interesting because of its small amplitude and rapid variabilities in both line and continuum fluxes. NGC 4151 is the brightest Seyfert 1 galaxy with an apparent magnitude of 11.2 and important for comparing the variabilities in other active galaxies monitored closely by IUE, such as 3C 273 (Ulrich et al. 1980; Ramos et al. 1997), NGC 5548 (Ulrich et al. 1983; Marshall et al. 1997), Mrk 335 (Hyung et al. 2000), NGC 3516 (Voit et al. 1987), NGC 7469 (Wanders 1997, Nandra et al. 1998) and Fairall 9 (Clavel et al. 1989). NGC 4151 has been classified as Seyfert 1.5 galaxy by Osterbrock and Koski (1976) since the spectrum had strong narrow emission components for the permitted lines along with broad lines. NGC 4151 is the only temporally well-observed Seyfert 1 galaxy by IUE satellite exhibiting large and rapid amplitude flux variations other than Fairall-9 (Clavel et al. 1989) and NGC 7469 (Wanders 1997). IUE observations of active galaxies have proved to be very important for establishing the rapid variability in UV continuum covering 1100-3200 $\AA$ wavelength region and many strong UV emission lines for the first time. NGC 4151 is an intrinsically low-luminosity AGN. It is a low redshift $(z=0.00332$, de Vaucouleurs et al. 1991) active galaxy and hence relatively at closer distance of $13 \mathrm{Mpc}\left(H_{0}=75 \mathrm{~km} / \mathrm{s} / \mathrm{Mpc}\right)$. Therefore, NGC 4151 appears roughly two orders of magnitude brighter than another long-time monitored active galaxy MRK $335\left(d_{L}=103 \mathrm{Mpc}\right)$. The BEL region in NGC 4151 is roughly three times bigger than Mrk 335 (Arav et al. 1998). Near simultaneous intense monitoring observations of NGC 4151 have been carried out by ROSAT, ASCA and Compton Gamma Ray Observatory 
(CGRO) during the period November 30 to December 13,1993 to study the multiwavelength short-timescale characteristics.

\section{IUE observations, data and reduction}

The nucleus of NGC 4151 was observed by blind offset from a nearby bright star SAO62869 as this procedure results into a positioning error in the aperture by less than 1. During the exposures the same bright star was used for guiding the object. The offset slew was reported once every eight hours to re-centre the target in the aperture and to update the guide star's position since the spacecraft rolls to maintain optical positioning of the solar arrays. With this observational technique, NGC 4151 was observed by IUE for more than 125 days from 1978 June to 1989 January. Since NGC 4151 has intrinsically low luminosity, it varies rapidly and during these long years of observational period of IUE, it has passed through several low and high flux states. Ulrich et al. (1991) have attributed the near-simultaneous variations in UV/optical variations to the instabilities in the inner part of the accretion disk emitting maximally in the UV/EUV range. The disk irradiation by variable $\mathrm{X}$-ray source has also been considered as an alternative process for the simultaneous UV/optical variability. The C IV line maintains a high degree of symmetry during the decrease and increase states of NGC 4151. The narrow emission lines in AGN spectra do not appear to vary rapidly because the NLR extends over several hundreds of parsecs. In contrast, the broad emission line variations on time-scales of several days imply that the BLR is of the order of one or two light days or even less.

The success of large scale monitoring campaigns of IUE is due to high temporal campaign rates over extended periods of observations of a few Seyfert 1 galaxies like NGC 4151, MRK 335, NGC 5548, NGC 3516 and NGC 7469. The cornerstones of most of these campaigns have been the observations of IUE, as it has provided long periods of observations at precise intervals, accurate absolute flux levels, where the continuum and high ionization lines are more strongly variable in UV than in the optical spectrum. Most of the IUE campaigns have focused on nearly bright Seyfert 1 galaxies whose UV continua and emission lines were known previously to be strongly variable. The nearly zero-lag delay between the variations in the UV continuum windows is the most significant result of the IUE's long-term monitoring from 1978 to 1991. The time-lag between the UV and optical light curves has been found to be $\leq 4 \pm 3$ days. This provided an important constraint on the models of the continuum source (Crenshaw et al. 1996). These results are comparable to similar campaigns on NGC 5548 and NGC 3783 in which it was found that the lags for the high ionization lines He II $\lambda 1640, \mathrm{~N}$ V $\lambda 1240$ were also $\leq 4 \pm 3$ days. Further, with still higher sampling observations by IUE, HST and ground-based campaigns on NGC 5548, Korista et al. (1995)were able to determine that the lags were slightly less than two days. A possible explanation for these delays is that UV and optical continuum emissions are from relatively cooler material emitting X-rays closer to the central source (Courvoisier \& Clavel 1991; Suzy 1991; Krolik et al. 1991). Thus, it became another compelling reason to carry out IUE observations with still higher temporal resolutions to check this result on all bright Seyfert galaxies like NGC 4151 and to fully resolve the transfer function of the high ionization lines (Peterson 1993). In this paper, we present our results on the spectral analysis of IUE's most intense observations of NGC 4151 extending over nearly 15 days during December $1-15,1993$ by $F_{\text {var }}$ and $R_{\max }$ variability characterization as the contribution to the flux variability by the instrument and the background noise is minimized in these methods. During this period, NGC 4151 was observed through SWP (1150-1970 A) and LWP (1970-3300 ̊) cameras with large apertures $\left(10^{\prime \prime} \times 20^{\prime \prime}\right)$ in low dispersion $(\Delta \lambda=$ 5-8 $\AA$ ) mode. The standard observing strategy was to obtain alternate SWP and LWP exposures by reading and preparing one camera, while the other camera was exposing, resulting in a pair of spectra every one hour approximately. The typical exposure times were $20 \mathrm{~min}$ for the SWP and $10 \mathrm{~min}$ for the LWP images. During each day of continuous monitoring, the observations were interrupted for two hours as the Earth occulted the target.

We have extracted a total of 100 NEWSIPS processed spectral data including both SWP and LWP cameras from the MAST IUE-NED database. The IUE's low dispersion spectra have shown an increase in the signalto-noise ratio of $\sim 10-50 \%$ with NEWSIPS processing (Nichols \& Linsky 1996). The spectra were further converted into filename.fits from filename.mxlo using IUETOOLS and IRAF tasks. Actually IUE has obtained a total of about 205 SWP and 196 LWP spectra during the intense monitoring campaign by Crenshaw et al. (1996). However, in this paper, we are presenting line and continuum flux measurement from only 63 SWP and 25 LWP spectra from our total sample of 100 spectra as a result of restricting the spectral quality to snr $\geq 5$. Crenshaw et al. (1996) have considered all the 401 spectra for their $F_{\text {var }}$ characterization of NGC 
Table 1. Log of IUE observations of NGC 4251.

\begin{tabular}{|c|c|c|c|c|c|c|c|}
\hline Spectrum & J.D. Start & Date of obs. & U.T. & Spectrum & J.D. Start & Date of obs. & U.T. \\
\hline SWP49381 & 2449322.746 & 12-01-1993 & $5: 44: 16$ & LWP26954 & 2449328.556 & 12-07-1993 & $1: 16: 10$ \\
\hline SWP49382 & 2449322.794 & 12-01-1993 & $6: 54: 02$ & SWP49494 & 2449328.666 & 12-07-1993 & $3: 48: 08$ \\
\hline SWP49392 & 2449323.344 & 12-01-1993 & $20: 05: 43$ & SWP49496 & 2449328.75 & 12-07-1993 & $5: 49: 52$ \\
\hline SWP49393 & 2449323.387 & 12-01-1993 & 21:07:48 & SWP49497 & 2449328.793 & 12-07-1993 & $6: 51: 00$ \\
\hline LWP26861 & 2449323.552 & $12-02-1993$ & 1:10:08 & SWP49498 & 2449328.84 & 12-07-1993 & $7: 58: 49$ \\
\hline SWP49395 & 2449323.578 & $12-02-1993$ & $1: 41: 56$ & SWP49507 & 2449329.254 & 12-07-1993 & $17: 56: 42$ \\
\hline SWP49397 & 2449323.678 & 12-02-1993 & 4:06:15 & LWP26971 & 2449329.322 & 12-07-1993 & 19:39:00 \\
\hline LWP26864 & 2449323.699 & 12-02-1993 & $4: 41: 27$ & SWP49509 & 2449329.346 & 12-07-1993 & 20:09:02 \\
\hline SWP49398 & 2449323.727 & $12-02-1993$ & $5: 17: 21$ & SWP49514 & 2449329.614 & $12-08-1993$ & $2: 34: 04$ \\
\hline SWP49399 & 2449323.777 & $12-02-1993$ & $6: 29: 06$ & SWP49516 & 2449329.698 & $12-08-1993$ & $4: 35: 52$ \\
\hline SWP49400 & 2449323.821 & 12-02-1993 & $7: 32: 56$ & LWP26978 & 2449329.719 & 12-08-1993 & $5: 10: 48$ \\
\hline SWP49409 & 2449324.294 & $12-02-1993$ & $18: 53: 34$ & SWP49518 & 2449329.784 & $12-08-1993$ & $6: 39: 15$ \\
\hline SWP49412 & 2449324.423 & $12-02-1993$ & $21: 58: 54$ & LWP26980 & 2449329.803 & $12-08-1993$ & $7: 11: 39$ \\
\hline LWP26880 & 2449324.581 & 12-03-1993 & 1:52:08 & SWP49519 & 2449329.833 & 12-08-1993 & $7: 49: 13$ \\
\hline SWP49415 & 2449324.651 & 12-03-1993 & $3: 28: 00$ & SWP49527 & 2449330.211 & 12-08-1993 & $16: 54: 10$ \\
\hline SWP49416 & 2449324.695 & 12-03-1993 & 4:31:01 & LWP26989 & 2449330.228 & 12-08-1993 & $17: 23: 20$ \\
\hline SWP49418 & 2449324.785 & 12-03-1993 & $6: 39: 48$ & LWP26992 & 2449330.367 & $12-08-1993$ & $20: 43: 49$ \\
\hline SWP49419 & 2449324.839 & 12-03-1993 & $7: 58: 12$ & LWP26993 & 2449330.411 & $12-08-1993$ & $21: 46: 46$ \\
\hline LWP26893 & 2449325.23 & 12-03-1993 & $17: 25: 31$ & SWP49533 & 2449330.529 & 12-09-1993 & $0: 31: 51$ \\
\hline LWP26896 & 2449325.372 & 12-03-1993 & $20: 50: 35$ & SWP49534 & 2449330.567 & 12-09-1993 & $1: 27: 21$ \\
\hline SWP49431 & 2449325.543 & 12-04-1993 & $0: 52: 25$ & SWP49538 & 2449330.746 & 12-09-1993 & $5: 45: 08$ \\
\hline SWP49432 & 2449325.585 & 12-04-1993 & $1: 52: 29$ & SWP49539 & 2449330.79 & 12-09-1993 & $6: 47: 53$ \\
\hline LWP26899 & 2449325.605 & 12-04-1993 & $2: 26: 16$ & SWP49547 & 2449331.214 & 12-09-1993 & $16: 56: 54$ \\
\hline LWP26900 & 2449325.651 & 12-04-1993 & $3: 32: 35$ & LWP27008 & 2449331.231 & 12-09-1993 & 17:27:06 \\
\hline SWP49434 & 2449325.675 & 12-04-1993 & $4: 02: 52$ & SWP49548 & 2449331.255 & 12-09-1993 & $17: 56: 41$ \\
\hline SWP49435 & 2449325.718 & 12-04-1993 & $5: 04: 28$ & LWP27010 & 2449331.321 & 12-09-1993 & 19:38:06 \\
\hline SWP49446 & 2449326.255 & 12-04-1993 & $17: 57: 50$ & SWP49550 & 2449331.346 & 12-09-1993 & 20:08:02 \\
\hline SWP49447 & 2449326.299 & 12-04-1993 & 19:00:19 & SWP49551 & 2449331.386 & 12-09-1993 & $21: 05: 29$ \\
\hline LWP26915 & 2449326.364 & 12-04-1993 & $20: 40: 35$ & SWP49554 & 2449331.559 & 12-10-1993 & $1: 14: 37$ \\
\hline SWP49450 & 2449326.546 & $12-05-1993$ & 0:57:09 & SWP49555 & 2449331.604 & $12-10-1993$ & 2:19:19 \\
\hline SWP49453 & 2449326.672 & $12-05-1993$ & $3: 58: 31$ & LWP27015 & 2449331.624 & 12-10-1993 & $2: 54: 11$ \\
\hline SWP49454 & 2449326.714 & 12-05-1993 & 4:59:49 & SWP49557 & 2449331.693 & 12-10-1993 & $4: 27: 56$ \\
\hline SWP49455 & 2449326.757 & $12-05-1993$ & $6: 00: 24$ & SWP49558 & 2449331.738 & $12-10-1993$ & $5: 33: 43$ \\
\hline SWP49464 & 2449327.225 & $12-05-1993$ & $17: 15: 18$ & SWP49560 & 2449331.853 & 12-10-1993 & $8: 18: 41$ \\
\hline LWP26931 & 2449327.242 & $12-05-1993$ & $17: 44: 15$ & SWP49567 & 2449332.755 & 12-11-1993 & $5: 57: 11$ \\
\hline SWP49466 & 2449327.32 & $12-05-1993$ & $19: 32: 32$ & LWP27025 & 2449332.825 & $12-11-1993$ & $7: 42: 12$ \\
\hline SWP49470 & 2449327.535 & 12-06-1993 & $0: 41: 47$ & SWP49582 & 2449334.751 & $13-12-1993$ & $5: 50: 03$ \\
\hline SWP49472 & 2449327.62 & 12-06-1993 & $2: 44: 03$ & LWP27034 & 2449334.768 & 13-12-1993 & $6: 18: 55$ \\
\hline LWP26937 & 2449327.64 & 12-06-1993 & $3: 16: 57$ & SWP49583 & 2449334.792 & 13-12-1993 & $6: 49: 05$ \\
\hline SWP49474 & 2449327.706 & 12-06-1993 & $4: 48: 10$ & LWP27035 & 2449334.81 & 13-12-1993 & $7: 20: 59$ \\
\hline SWP49475 & 2449327.751 & 12-06-1993 & $5: 52: 24$ & SWP49592 & 2449335.746 & 14-12-1993 & $5: 42: 29$ \\
\hline SWP49476 & 2449327.794 & 12-06-1993 & $6: 53: 05$ & LWP27041 & 2449335.806 & 14-12-1993 & $7: 14: 49$ \\
\hline SWP49486 & 2449328.273 & 12-06-1993 & $18: 23: 43$ & LWP27048 & 2449336.767 & $15-12-1993$ & $6: 18: 25$ \\
\hline SWP49487 & 2449328.318 & 12-06-1993 & $19: 28: 24$ & SWP49601 & 2449336.791 & $15-12-1993$ & $6: 46: 47$ \\
\hline
\end{tabular}

4151. The spectra considered for the variability characterization studies are of better quality compared to Crenshaw et al. (1996). Observational details of the intense monitoring campaign for over 15 days are given in Table 1. The effects of reddening by dust on the UV continuum and emission line fluxes can be very significant. But since our results are fractional amplitudes and have short time scale variations, the results of analysis 
would not be affected by galactic reddening. Galactic reddening is essentially considered zero since the HI column density estimated to be $N_{\mathrm{HI}} \sim 2.1 \times 10^{20} \mathrm{~cm}^{-2}$ by Stark et al. (1992) yields $E(B-V) \leq 0.01$ (Burstein $\&$ Heiles 1982). Penston et al. (1981) have shown that the total reddening of the UV continuum as determined from the $2200 \AA$ feature is $E(B-V)=0.1$ and Crenshaw et al. (1996) have verified this result by using the average NGC 4151 spectrum of the present campaign and assuming that the interstellar extinction curve of Savage and Mathis (1979) is applicable. Kriss et al. (1992) have calculated $E(B-V)=0.039$ from a power law plus galactic extinction fit to the far UV spectrum of NGC 4151. For the narrow emission lines, Kriss et al. (1992) determined that $E(B-V)=0.12$ from the recombination lines He II $\lambda 1085 \AA$ and He II $\lambda 1640 \AA$. Thus there is currently no accurate way to determine the reddening of the broad components of the lines although it is not expected that dust could survive in the broad line region of NGC 4151 (Ferland \& Mushotzky 1982). In this study, all spectra have been de-reddened using $E(B-V)=0.032$. The reddening corrected spectra were further reduced for redshift effect by taking the accurate optical redshift of $z=0.00332$ and using the dopcor task under IRAF to facilitate flux measurements at the object's rest-frame wavelengths.

\section{Measurements of continuum, line fluxes and variability characterization}

The continuum fluxes have been measured at line free regions in both SWP and LWP spectra with a constant window of $50 \AA$ centred at $1325,1475,1655,2175$, 2425 and $2725 \AA$. The continuum fluxes are weighted means over the band pass and the errors in the continuum fluxes are 1 sigma standard deviations of the mean over the $50 \AA$ band pass. This is similar to the procedure adopted by Gondhalekar (1990) for studying the continuum variability in 34 quasars observed by IUE. Fluxes of strong emission lines namely $\mathrm{Ly} \alpha, \mathrm{Si}$ IV, C IV, He II, C III] and Mg II were measured by fitting single Gaussian profile by visual inspection method for the local continuum. The flux of the emission lines was measured from the mean continuum level on either side of an emission line. The C IV emission line has three features: blue wing, the central absorption line and a red wing. The absorption line has been observed to be asymmetric throughout the observational period. Asymmetric blue and red wings of C IV line indicate the presence of radial motions and/or a clumpy non symmetrical distributions of clouds (Ulrich et al. 1991). The

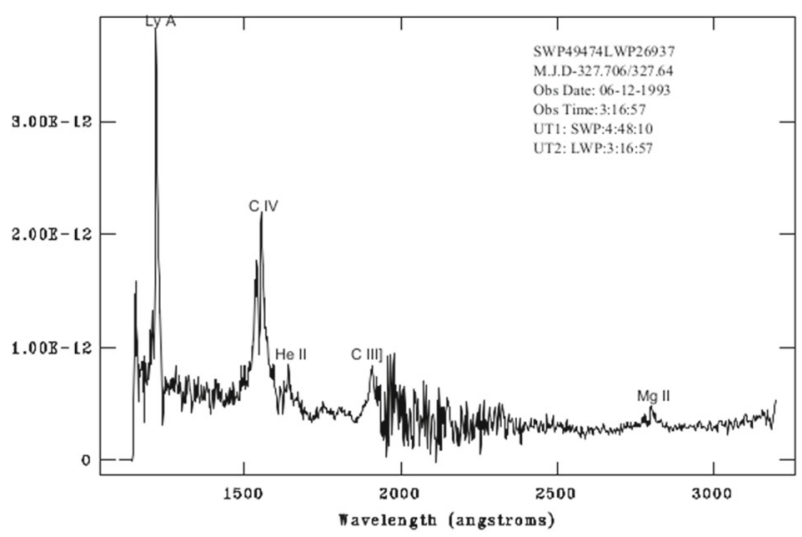

Figure 1. Long combined spectrum corresponding to the highest flux state of the intense monitoring campaign. The two spectra of SWP49474 and LW26P937 are simultaneous within a time interval of $1.5 \mathrm{~h}$.

flux of absorption line intercepting C IV emission line was also measured by fitting a single Gaussian profile by simply connecting two base points by a horizontal straight line. The line fluxes of C IV were measured by removing the absorption feature using IRAF tasks, similar to that of Ulrich et al. (1991). The blue-shifted C IV absorption line feature appearing at the centre of the $\mathrm{C}$ IV emission line has also exhibited variability and results of the same would be presented in our next paper along with our similar study on NGC 7469. The combined short wavelength and long wavelength spectra for the highest flux state of the campaign is given in Fig. 1. The wavelength region between $1950-2350 \AA$ is very noisy because of the poor sensitivity of the long wavelength camera. The continuum and emission line light curves are presented in Fig. 2. The variability in continuum and line flux are generally characterized by ratio of the fluxes measured $\left(R_{\max }=F_{1} / F_{2}\right)$ corresponding to high and low flux states of an observational period as this would be useful in quantifying the variability amplitude directly from the spectrum, independent of theoretical models. The continuum and line flux variabilities in active galaxies are usually characterized by several parameters. The three parameters widely used are the mean flux, the rms flux and the relative variability amplitude defined as follows:

$$
\begin{aligned}
\bar{F}_{\lambda} & =\frac{1}{N} \Sigma_{i=1}^{N} F_{\lambda i}, \\
\sigma_{F_{\lambda}}^{2} & =\frac{1}{N-1} \Sigma_{i=1}^{N}\left(F_{\lambda i}-\bar{F}_{\lambda}\right)^{2}, \\
R_{\max } & =\frac{F_{\lambda 1}}{F_{\lambda 1}} .
\end{aligned}
$$




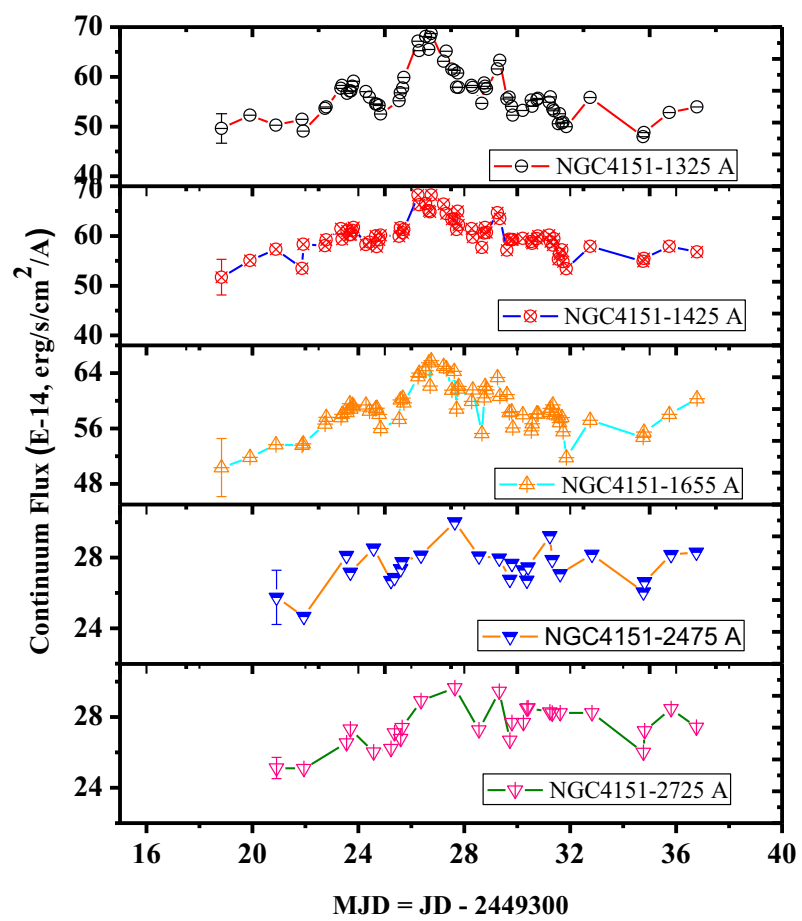

Figure 2. Continuum light curves of NGC 4151 for the intense monitoring campaign of IUE during December 1-15, 1993.

A fourth parameter, defined as $F_{\text {var }}$ is considered as an estimate of the amplitude of the intrinsic variability relative to the mean flux according to Vaughan et al. (2003) and corrected for the measurement errors $\Delta F_{\lambda i}$ and is defined as

$F_{\mathrm{var}}=\frac{1}{\bar{F}_{\lambda}} \sqrt{\left(\sigma_{F_{\lambda}}^{2}-\Delta \bar{F}_{\lambda}^{2}\right)}$

where $\Delta \bar{F}_{\lambda}^{2}$ the mean square of the flux measurement uncertainities. The $\Delta \bar{F}_{\lambda}^{2}$ is further defined as

$\Delta \bar{F}_{\lambda}^{2}=\frac{1}{N} \Sigma_{i=1}^{N} \Delta F_{\lambda i}^{2}$.

The $F_{\text {var }}$ parameter characterizes the variability amplitude by taking the measurement errors into account. Alternatively, $F_{\text {var }}$ is also called as Normalized Variability Amplitude (NVA) according to Edelson et al. (1996)and make use of the mean flux, standard deviation of the measured flux points and the mean error in the fluxes. $F_{\mathrm{var}}$ or $\sigma_{\mathrm{NVA}}$ is supposed to be free of instrumental effects as it is estimated by subtracting in quadrature the measured mean error in the measured fluxes from the standard deviation and divided by the mean flux. This analysis is essentially the same procedure that was used to derive $F_{\mathrm{var}}$ by Peterson et al. (1991) and $\sigma_{\mathrm{NVA}}$ by Edelson
(1996). A summary of these variability characteristics obtained in the present analysis are given in Table 2. For comparison, we have also provided the results obtained by Crenshaw et al. (1996) and Edelson et al. (1996) in Table 2.

\section{Results, discussions and conclusions}

From the continuum light curves shown in Fig. 2, we see that there are four clearly distinguishable significant events of small and rapid amplitude variations within an interval of 4-5 days. The amplitudes of variability in active galaxies are often characterized by the relative amplitude parameter $R_{\max }$. We have found $R_{\max }$ values to be $1.372,1.319,1.302$ and 1.182 for continuum at $1325,1475,1655$ and $2725 \AA$ respectively. Crenshaw et al. (1996) have obtained the $R_{\max }$ values to be $1.51,1.45,1.31$ and 1.24 for the continuum wavelengths at $1275,1440,1820$ and $2688 \AA$ respectively with more number of spectra included in the analysis. Our relative variability amplitude parameters obtained in the present analysis are in perfect agreement with those of Crenshaw et al. (1996) despite our different methodology of continuum flux measurements and the reddening parameter adopted. Thus, we clearly see that the amplitude of variability has decreased with increasing wavelength. Such a behaviour has also been seen in the other long-term monitoring campaigns on NGC 5548 by Clavel et al. (1991) and Korista et al. (1995), and on NGC 3783 by Riechert et al. (1994).

The UV continuum variability data presented in this paper are simultaneous within a time interval of about an hour approximately and closest-time interval monitoring by the IUE. The observational temporal resolution of limit of $\Delta t \sim 1 \mathrm{~h}$ simultaneity in the UV region on NGC 4151 thus provides a strict constraint on time-delays compared to the upper limits on $\mathrm{UV}$ lags of $\Delta t \sim 4 \pm 3$ days for $\mathrm{C}$ IV line (Clavel et al. 1990) and $9 \pm 2$ days for Balmer lines (Maoz et al. 1991) in NGC 4151. The peak fluxes of the four events obtained in the present study are however small compared to the results of the previous longer time-scale campaigns on NGC 4151 (Clavel et al. 1990) and are significantly large compared to the errors in the continuum fluxes (since $\mathrm{snr} \geq 5$ ) demonstrating the suitability and the importance of IUEs continuous monitoring of Seyfert 1 galaxies with higher temporal resolution. We also see subpeaks of smaller amplitude variability within the four major events, but are difficult to be identified due to 
Table 2. Continuum and line flux variability characteristics.

\begin{tabular}{|c|c|c|c|c|c|c|}
\hline $\begin{array}{l}\text { Continuum/ } \\
\text { line feature }\end{array}$ & $\begin{array}{c}\text { No. of } \\
\text { data points }\end{array}$ & $\begin{array}{l}\text { Mean } \\
\text { flux }\end{array}$ & $\begin{array}{l}\text { Mean } \\
\text { error }\end{array}$ & $\begin{array}{c}\text { Mean } \\
\sigma \text {-error }\end{array}$ & $\begin{array}{c}F- \\
\text { variance }\end{array}$ & $R_{\max }$ \\
\hline $50-150 \mathrm{KeV}^{\mathrm{b}}$ & 9 & - & - & - & 0.061 & - \\
\hline $1-2 \mathrm{KeV}^{\mathrm{b}}$ & 17 & - & - & - & 0.239 & - \\
\hline $1275 \AA^{\mathrm{a}}$ & 176 & 43.45 & 0.9 & - & 0.091 & 1.51 \\
\hline $1275 \AA^{b}$ & 176 & - & - & - & 0.086 & - \\
\hline $1325 \AA$ & 63 & 57.037 & 8.074 & 8.192 & 0.083 & 1.372 \\
\hline $1330 \AA ̊$ & 176 & - & - & - & 0.082 & - \\
\hline $1440 \AA^{\mathrm{a}}$ & 176 & 37.66 & 1.5 & - & 0.07 & 1.45 \\
\hline $1440 \AA^{b}$ & - & - & - & - & 0.062 & - \\
\hline $1475 \AA$ & 63 & 60.371 & 8.989 & 8.989 & 0.053 & 1.319 \\
\hline $1655 \AA$ & 63 & 59.366 & 8.903 & 8.903 & 0.048 & 1.302 \\
\hline $1730 \AA^{b}$ & - & - & - & - & 0.06 & - \\
\hline $1820 \AA^{\mathrm{a}}$ & 176 & 28.83 & 1.3 & - & 0.052 & 1.31 \\
\hline $1820 \AA^{b}$ & - & - & - & - & 0.048 & - \\
\hline $1950 \AA^{b}$ & - & - & - & - & 0.045 & - \\
\hline $2300 \AA^{b}$ & - & - & - & - & 0.049 & - \\
\hline $2725 \AA$ & 24 & 27.658 & 2.211 & 2.255 & 0.037 & 1.24 \\
\hline $6200 \AA^{b}$ & - & - & - & - & 0.014 & - \\
\hline $6925 \AA^{b}$ & - & - & - & - & 0.14 & - \\
\hline C IV & 63 & 65.063 & 0.132 & 0.132 & 0.034 & 1.23 \\
\hline $\mathrm{CIV}^{\mathrm{a}}$ & - & 45.95 & 0.014 & - & 0.054 & 1.33 \\
\hline He II & - & 6.414 & 0.068 & 0.096 & 0.177 & 3.052 \\
\hline $\mathrm{He} \mathrm{II}^{\mathrm{a}}$ & 63 & 6.54 & 0.042 & - & 0.077 & 1.7 \\
\hline C III] & 63 & 11.336 & 0.052 & 0.052 & 0.112 & 1.975 \\
\hline $\mathrm{C} \mathrm{III]}]^{\mathrm{a}}$ & - & 6.54 & 0.034 & - & 0.077 & 1.49 \\
\hline Mg II & 23 & 10.786 & 0.024 & 0.024 & 0.115 & 1.55 \\
\hline
\end{tabular}

The continuum fluxes are in units of $10^{-14} \mathrm{erg} / \mathrm{s} / \mathrm{cm}^{2} / \AA$; line fluxes are in $10^{-12} \mathrm{erg} / \mathrm{s} / \mathrm{cm}^{2}$; ${ }^{\mathrm{a}}$ Crenshaw et al. (1996) and ${ }^{\mathrm{b}}$ Edelson et al. (1996).

the background UV noise. Future observations with still higher temporal resolution, spectral resolution and sensitivity would resolve the features within these smaller amplitude variations. Currently, variability in active galaxies is frequently characterized by $F_{\text {var }}$ analysis as discussed in section 3 . The results of the $F_{\text {var }}$ analysis parameters are summarized in Table 1 . For our continuum and line flux measurements, we have obtained highest $F_{\text {var }}$ of nearly $8.3 \%$ at $1352 \AA$ continuum with a smallest amplitude of $\sim 4 \%$ at $2725 \AA$ comparable to the results obtained by Crenshaw et al. (1996) and Edelson et al. (1996). Thus, we observe a trend of decrease in the $F_{\text {var }}$ parameter for the continuum fluxes with increasing wavelengths indicating that the amplitude of continuum flux variations decreases with increasing wavelength. Similar results have been obtained by Clavel et al. (1991) in NGC 5548, Riechert et al. (1994) in NGC
3783 for both the $F_{\text {var }}$ and $R_{\max }$ parameters. These results are in general agreement with the previous IUE studies of NGC 4151 by Perola et al. (1982) which found that the spectrum at longer wavelengths becomes harder when the flux increases. The trend of decreasing amplitude with respect to increasing wavelength obtained in the present analysis along with the strikingly similar results by Crenshaw et al. (1996) and Edelson et al. (1996) provides a significant constraint on the UV continuum variability amplitude in NGC 4151, which is archetypal of Seyfert 1 galaxies particularly, and active galaxies, in general. NGC 4151 is well-studied for short timescale variability across $\mathrm{X}$ rays/UV/optical wavebands by presently in-orbit space observatories such as Swift and AstroSat. NGC 4151, a Seyfert 1.5 galaxy is typically the brightest Seyfert galaxy in the Universe at X-rays/UV/optical wavelengths (Edelson et al. 2017). 
Krimm et al. (2013) through Swift observations have reported that the NGC 4151 is twice as bright in the $15-150 \mathrm{keV}$ band as the next brightest type 1 AGN. From the softest X-ray observations by ASCA, the flux of NGC 4151 was found to be constant throughout the observartional period during November 30 to December 13, 1993, while significant variations were observed by a factor of 1.45 in the count rate in $1.0-2.0 \mathrm{keV}$ within a timescale of two days. Similar to the results of IUE's intense observations presented in this paper and in contrast to the results of ROSAT, a low amplitude variability of $\leq 10 \%$ was observed from the simultaneous observations by ASCA in the $2-10 \mathrm{keV}$ band. The $0.1 \mathrm{keV}$ to $300 \mathrm{keV}$ spectrum of NGC 4151 was also found to be dominated by hard power-law continuum from these near simultaneous observations by ROSAT, ASCA and CGRO (Warwick et al. 1996). A high energy cut-off near $90 \mathrm{keV}$ is characteristic of continuum emission generated by the process of thermal Comptonization and the low energy cut-off would be due to the absorptions in the line-of-sight gas. The gamma ray count rates from OSSE spectrometer onboard CGRO satellite were found to be consistent with $\pm 15 \%$ in the $50-150 \mathrm{keV}$ band and has not provided any direct correspondence between gamma rays and soft X-rays from their light curves. However, Warwick et al. (1996) have reported a correlation between 1-2 keV X-ray and ultraviolet continuum at $1400 \AA$ with higher relative amplitude of variability at X-rays. The slope of the X-ray and UV correlation has been found to be similar to earlier studies. The four major events of flux maxima obtained shown in Fig. 2 are quite similar to that of Crenshaw et al. (1996) with an interval of 3-4 days. The flux rises slowly and drops off rapidly suggesting slow response timescales of emission line regions to the variable hard continuum radiation. It is further observed for the emission lines that the $F_{\mathrm{var}}$ is relatively larger and over estimates the strength of the fast intrinsic UV variations. The $F_{\text {var }}$ and the mean fluxes of C IV and C III] emission lines obtained by us are comparable to Crenshaw et al. (1996), while no comparison is available for our results on Mg II line. We see no good correlation between continuum and emission line variabilities over this short timescale observations. This indicates, in our opinion, that BLR takes longer response timescales to the fast continuum flux variations. More studies with intensive, long-term and high resolution UV spectral campaigns are required to re-confirm these results. We expect that such studies are possible with ASTROSAT observations and enable us to better understand the complex phenomenon of line and continuum variabilities in active galaxies.

\section{Acknowledgements}

The work presented in this paper has been carried out under the grants sanctioned to the second author through the Major Research Project Scheme of UGC, New Delhi. Hence, we acknowledge the financial support provided by UGC, New Delhi and Department of Physics, Bangalore University, Bengaluru for providing necessary infra structure facilities. We acknowledge the assistance of Dr Pandge, SRTM University, Nanded and Dr K. Drysya, Department of Physics, Bangalore University in the installation of IUETOOLS software and other related issues.

\section{References}

Arav N., Barlow T. A., Laor A., Sargent W. L. W., Blandford R. D. 1998, MNRAS, 297, 990

Bahcall J. N., Kozlovsky B., Salpeter E. E. 1972, Astrophys. J., 171, 467

Blandford R. D., McKee C. F. 1982, Astrophys. J., 225, 419

Boggess A., Carr F. A., Evans D. C. 1978a, Nature, 275, 372

Boggess A., Bohlin R. C., Evans D. C. 1978b, Nature, 275, 377

Burstein D., Heiles C. 1982, Astron. J., 87, 1165

Cherepashchuk A. M., Lyutyi V. M. 1973, Astrophys. Lett., 13,165

Clavel J., Riechert G. A., Alloin D. et al. 1991, Astrophys. J., 366, 64

Clavel J., Boksenberg A., Bromage G. E. et al. 1990, MNRAS, 246, 668

Clavel J, Wamsteker W., Glass I. S. 1989, Astrophys. J., 337, 236

Collin-Souffrin Suzy, 1991, A\&A, 249, 344

Courvoisier T. J.-L., Clavel J. 1991, A\&A, 248, 389

Crenshaw D. M., Rodriguez-Pascual Penton S. V. et al. 1996, Astrophys. J., 470, 322

de Vaucouleurs G., de Vaucouleurs A., Corwin H. G. Jr. et al. 1991 in Third Reference Catalog of Bright Galaxies (Berlin: Springer).

Edelson R. A., Alexander T., Crenshaw D. M. et al. 1996, Astrophys. J., 470, 364

Edelson R., Gelbord J., Cackett E. et al. 2017, Astrophys. J., 840,41

Ferland G. J., Mushotzky R. F. 1982, Astrophys. J., 262, 564

Gondhalekar P. M. 1990, MNRAS, 243, 443

Gondhalekar P. M., Kellet B. J. 1994, in Gondhalekar P. M., Horne K., Peterson B. M., eds, ASP Conference Series, vol. 69, Reverberation Mapping of the Broad Line Region in Active Galactic Nuclei, San Francisco

Gonzalez-Martin O., Vaughan S. 2012, A\&A, 544, A80

Hyung S., Kim H., Lee W. K., Lee S. J., Ryu D., Lee H. W. 2000. J. Korean. Astron. Soc., 33, 81

Korista K. T., Alloin D., Barr P. et al. 1995, ApJSS, 97, 285 
Krimm H. A., Holland S. T., Corbet R. H. D. 2013, ApJSS, 209,14

Kriss G. A., Davidsen A. F., Blair P. et al. 1992, Astrophys. J., 392, 485

Krolik J. H., Horne K., Kallman T. R., Malkan M. A., Edelson R. A., Kriss G. A. 1991, Astrophys. J., 371, 541

Marshall H. L., Carone T. E., Peterson B. M., Clavel J. 1997, Astrophys. J., 479, 222

Nandra K., Clavel J., Edelson R. A. et al. 1998, Astrophys. J., 505, 594

Netzer H. 1990, in Blandford R. D., Netzer H., Woltjer L., eds, Saas-Fee Advanced Course 20: Active Galactic Nuclei, Springer, New York, p. 57

Nichols J. S., Linsky J. L. 1996, Astron. J., 111, 517

Osterbrock D. E., Koski A. T. 1976, MNRAS, 176, 61

Penston M. V., Boksenberg A., Bromage G. E., et al. 1981, MNRAS, 196, 857

Perola G. C., Boksenberg A., Bromage G. E. et al. 1982, MNRAS, 200, 293

Peterson B. M. 1993, PASP, 105, 293

Peterson B. M., Balonek T. J., Barker E. S. et al. 1991, Astrophys. J., 368, 119
Ramos E., Kafatos M., Fruscione A., et al. 1997, Astrophys. J., 482, 167

Riechert G. A., Rodriguez-Pascual P. M., Alloin D. et al. 1994, Astrophys. J., 425, 582

Savage B. D., Mathis J. S. 1979, ARAA, 17, 73

Stark A. A., Grammie C. F., Wilson R. W., Bally J., Linke R. A., Heiles C., Hurwitz M. 1992, ApJSS, 79, 77

Ulrich M. H., Boksenberg A., Bromage G. E., Carswell R. et al. 1980, MNRAS, 192, 561

Ulrich M. H., Boisson C. 1983, Astrophys. J., 267, 515

Ulrich M. H., Boksenberg A., Bromage G. E., Clavel J., Elvius A., Penston M. V., Perola G. C., Snidjers M. A. J. 1991, Astrophys. J., 382, 483

Ulrich M. H., Horne K. 1996, MNRAS, 283, 748

Vaughan S., Edelson R., Warwick R. S., Uttley P. 2003, MNRAS, 345, 1271

Voit M., Shull J. M., Begelman M. C. 1987, Astrophys. J., 316, 573

Wanders I., Peterson B. M., Alloin D., Ayres T. R. et al. 1997, ApJSS, 113, 69

Warwick R. S., Smith D. A., Yaqoob T., Edelson R., et al. 1996, Astrophys. J., 470, 349 